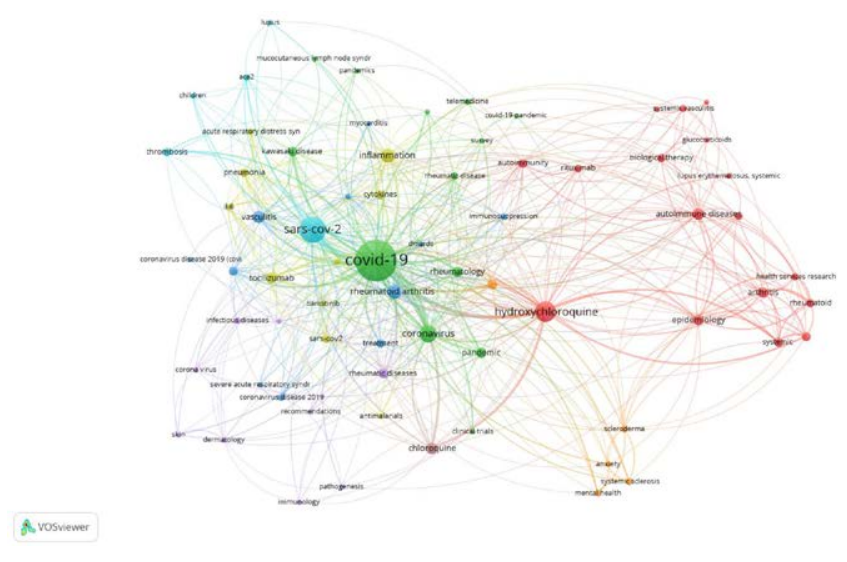

Figure 1.

Results: A total of 844 literature items were obtained. After screening of title, abstract and keywords we excluded 106 records as they were not emphasized the rheumatological perspective on COVID-19 and as a result were inapplicable for this study. The 738 retrieved articles were mostly (86.8\%) open access publications. The top five journals that contributed most to the coverage of this topic were: Annals Of The Rheumatic Diseases $(n=59)$, Clinical Rheumatology $(n=41)$, Lancet Rheumatology $(n=24)$, Arthritis And Rheumatology $(n=20)$ and Rheumatology International $(n=19)$. The origin of most studies was not surprisingly from those countries, which belong to the top ten according to the total cases of COVID-19 [1] (USA - 167; Italy - 148; UK - 76; India - 60 and Spain - 58). Most items were written in English but articles in German $(n=12)$, Spanish $(n=11)$, Russian $(n=5)$ and Chinese $(n=2)$ could also be found. Analyzed studies were designed in the form of Original Articles (41.2\%), Reviews (23.7\%), Letters (21.8), Notes $(6.9 \%)$, Editorials $(5.1 \%)$. According to the citations scores, articles of highest interest were dedicated to clinical course of COVID-19 in patients with autoimmune pathologies. The other highly cited studies were about cytokine storm and perspective usage of biological drugs for severe cases of COVID19. Our analysis of keywords showed that the most widely discussed rheumatic disease in the view of COVID-19 was systemic lupus erythematosus $(n=188)$, followed by vasculitis $(n=132)$, rheumatoid arthritis $(n=90)$, systemic sclerosis $(\mathrm{n}=32)$ and psoriatic arthritis (24). The liveliest discussion about disease-modifying antirheumatic drugs in COVID-19 revolved around hydroxychloroquine $(n=305)$, corticosteroids $(n=161)$, tocilizumab $(n=83)$, methotrexate $(n=46)$ and anakinra $(n=34)$.

Conclusion: As far as we know, it is the first bibliometric overview of studies dedicated to interrelation between COVID-19 and rheumatic pathology. The high number of open access items contributes to the increase of research visibility in this emergently developing research field and facilitates the process of scientific data sharing. The conducting of bibliographic studies may provide a valuable guide through this area of knowledge.

REFERENCES:

[1] https://www.worldometers.info/coronavirus/ Accessed on January 12, 2021

Disclosure of Interests: None declared.

DOI: 10.1136/annrheumdis-2021-eular.734

\section{POS1173 A SHORT DELAY IN ARTHRITIS CARE IS ASSOCIATED WITH HIGHER DISEASE ACTIVITY - LESSONS FROM THE CORONAVIRUS DISEASE 19 (COVID-19) PANDEMIC}

A. Giollo ${ }^{1}$, E. Bertoldo ${ }^{1}$, C. Benini ${ }^{1}$, O. Viapiana ${ }^{1}$, L. Idolazzi ${ }^{1}$, G. Adami ${ }^{1}$ A. Fassio ${ }^{1}$, D. Gatti ${ }^{1}$, M. Rossini ${ }^{1} .{ }^{1}$ University of Verona, Rheumatology Section, Department of Medicine, Verona, Italy

Background: Although disease activity is a significant outcome in rheumatology, few studies have investigated the relationship between routine care of rheumatic conditions and disease activity control.

Objectives: To determine the association between delay in routine care of chronic inflammatory arthritides (CIAs) and disease activity during the first wave of coronavirus disease 19 pandemic in Verona, Italy.

Methods: This study enrolled patients with an established diagnosis of rheumatoid arthritis (RA), psoriatic arthritis (PsA), ankylosing spondylitis (AS) and undifferentiated arthritis (UA). Between 01/04/2020 and 30/06/2020, participants were emailed an online questionnaire. Items comprised multiple or single-choice questions evaluating routine rheumatology care disruptions/delays and disease characteristics, treatments, comorbidities, and demographics. Compliance to anti-rheumatic medications was evaluated with I-CQR5. Disease activity was assessed with RAPID3, and active disease was defined as a RAPID3 score>1. Study period referred to the time between the last rheumatology assessment and the date of enrolment.

Results: Of 1210 patients contacted, 450 participated, of whom $219 \mathrm{CIAs}$ patients were included (RA 55.3\%, PsA 35.2\%, AS 15.1\%, UA 3.7\%). One hundred twenty-five patients $(57.1 \%)$ had their routine clinical assessment delayed (median days 68.4; IQR 66.8, 85.9). Patients in this group had significantly higher MDHAQ $(p=0.001)$ and RAPID3 ( $p=0.031)$ scores, while they did not differ for disease severity, medications or compliance. Most (87.7\%) reported good compliance to therapy; only $5.9 \%$ had difficulties in supplying anti-rheumatic medications, and $13.2 \%$ discontinued medications for at least four weeks for any reason. However, several patients $(37.9 \%)$ reported moderate-to-high worse disease activity per ception due to routine care delay, and $31.1 \%$ self-reported a disease flare (median RAPID3 score 3.8; IQR 2.0, 5.4). One hundred one patients $(46.1 \%$ ) had high disease activity, while only $15.1 \%$ were in remission. In logistic regression, active disease was significantly associated with delay of scheduled routine care visit independent of disease duration, time from last rheumatology assessment, therapy with b/tsDMARDs, and compliance (Table 1, Figure 1 below).

Table 1.

\begin{tabular}{|c|c|c|c|c|c|c|}
\hline Predictor & $\begin{array}{c}\text { Active } \\
(n=186)\end{array}$ & $\begin{array}{c}\text { Remission } \\
(n=33)\end{array}$ & OR & P-value & $\begin{array}{l}95 \% \mathrm{Cl} \\
\text { Inf. }\end{array}$ & $\begin{array}{l}95 \% \mathrm{Cl} \\
\text { Sup. }\end{array}$ \\
\hline Routine care delay (yes vs no) & $113(60.8 \%)$ & $12(36.4 \%)$ & .030 & 2.459 & 1.091 & 5.543 \\
\hline Disease duration (years) & $\begin{array}{c}10 \\
(4.0,20.0)\end{array}$ & $\begin{array}{c}10.5 \\
(7.0,19.8)\end{array}$ & .695 & 1.007 & .972 & 1.044 \\
\hline $\begin{array}{l}\text { Time since last rheumatology } \\
\text { assessment (days) }\end{array}$ & $\begin{array}{c}68.4 \\
(66.8,75.8)\end{array}$ & $\begin{array}{c}71.2 \\
(66.9,74.9)\end{array}$ & .854 & 1.002 & .978 & 1.027 \\
\hline b/tsDMARDs (yes vs no) & $\begin{array}{c}105 \\
(56.5 \%)\end{array}$ & $\begin{array}{c}15 \\
(45.5 \%)\end{array}$ & .653 & 1.202 & .538 & 2.687 \\
\hline $\begin{array}{l}\text { I-CPR5 (likely low vs likely high } \\
\text { adherent) }\end{array}$ & $\begin{array}{c}36 \\
(19.4 \%)\end{array}$ & $\begin{array}{c}10 \\
(30.3 \%)\end{array}$ & .187 & .563 & .240 & 1.321 \\
\hline
\end{tabular}

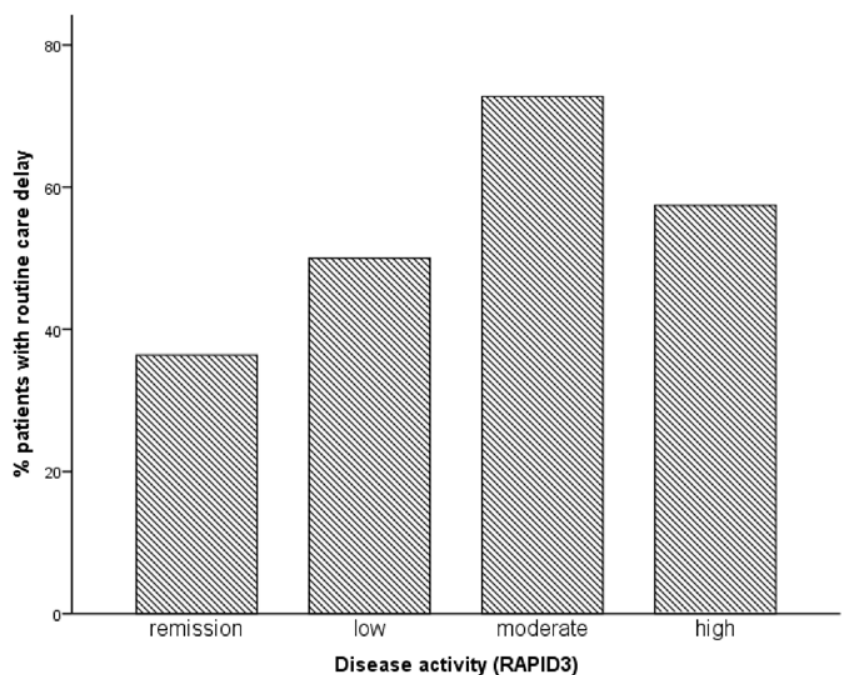

Figure 1.

Conclusion: In patients with established CIAs, a relatively short delay in routine assessment by a rheumatologist resulted in higher disease activity. Frequent rheumatology referrals appear to be a critical factor for disease activity control in CIAs. Disclosure of Interests: None declared.

DOI: 10.1136/annrheumdis-2021-eular.737

\section{POS1174 HYPERINFLAMMATION AND CLINICAL OUTCOMES FOR PATIENTS WITH SYSTEMIC RHEUMATIC DISEASES HOSPITALIZED FOR COVID-19: A COMPARATIVE COHORT STUDY}

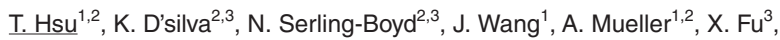
L. Prisco ${ }^{1}$, L. Martin ${ }^{1}$, K. Vanni ${ }^{1}$, A. Zaccardelli ${ }^{1}$, C. Cook $^{3}$, H. Choi ${ }^{2,3}$, Y. Zhang ${ }^{2,3}$, E. Gravallese ${ }^{1,2}$, Z. Wallace ${ }^{2,3}$, J. Sparks ${ }^{1,2} .{ }^{1}$ Brigham and Women's Hospital, Division of Rheumatology, Inflammation, and Immunity, Boston, United States of America; ${ }^{2}$ Harvard Medical School, Division of Rheumatology, Inflammation, and Immunity, Boston, United States of America; ${ }^{3}$ Massachusetts General Hospital, Division of Rheumatology, Allergy, and Immunology, Boston, United States of America 
Background: COVID-19 can induce a hyperinflammatory state resulting in cytokine storm, which can lead to poor outcomes. Patients with systemic rheumatic diseases may be at increased risk for respiratory failure with COVID-19. Therefore, we investigated the relationship between rheumatic disease, hyperinflammation, and clinical outcomes among hospitalized COVID-19 patients.

Objectives: To compare laboratory values, hyperinflammation, and clinical outcomes of hospitalized COVID-19 rheumatic patients and matched comparators.

Methods: We performed a comparative cohort study of patients with polymerase chain reaction (PCR)-confirmed COVID-19 requiring hospitalization between $3 / 1 / 20-7 / 7 / 20$ at a large health care system. We compared each systemic rheumatic disease case to up to 5 matched (by age, sex, and date of +SARS-CoV-2 PCR) comparators without systemic rheumatic disease. We extracted laboratory values from their hospitalization to compare peaks/troughs of individual laboratory results by case status and derived the COVID-19-associated hyperinflammation score (cHIS), a composite of 6 laboratory domains $(0-6, \geq 2$ indicating hyperinflammation), as previously developed ${ }^{1}$. We used multivariable logistic regression to estimate ORs for COVID-19 outcomes by hyperinflammation and case status.

Results: We identified 57 hospitalized rheumatic disease cases (mean age 67 years, 67\% female) and 232 matched comparators hospitalized with PCR-confirmed COVID-19. Among cases, 26 (46\%) had rheumatoid arthritis and 14 $(25 \%)$ had systemic lupus erythematosus. Most cases $(34,60 \%)$ had active rheumatic disease. At baseline, $15(27 \%)$ of cases were treated with biologic DMARDs, and 32 (56\%) were using glucocorticoids. We analyzed 39,900 total laboratory results (median 85 per patient). Cases had higher peak neutrophil-to-lymphocyte ratio ( 9.6 vs $7.8, p=0.02$ ), $\mathrm{LDH}$ (421 vs $345 \mathrm{U} / \mathrm{L}, p=0.04$ ), creatinine (1.2 vs $1.0 \mathrm{mg} / \mathrm{dL}, p=0.01$ ), and BUN (31 vs $23 \mathrm{mg} / \mathrm{dL}, p=0.03$ ) than comparators but similar peak CRP (149 vs $116 \mathrm{mg} / \mathrm{L}, p=0.11$, Figure 1 ). Cases had higher peak median cHIS ( 3 vs $2, p=0.01$ ). Peak $\mathrm{cHIS} \geq 2$ had higher odds of intensive care unit (ICU) admission (OR 3.45, 95\%Cl 1.98-5.99), mechanical ventilation (OR $66.0,95 \% \mathrm{Cl} 9.0-487.8$ ), and mortality (OR $16.4,95 \% \mathrm{Cl}$ 4.8-56.4) compared to cHIS <2 (Table 1). Cases had increased risk of ICU admission (OR 2.0, 95\% Cl 1.1-3.7) and mechanical ventilation (OR 2.7, 95\% Cl 1.4-5.2) than comparators

Table 1. Associations of peak cHIS and systemic rheumatic disease with COVID-19 hospitalization outcomes

\begin{tabular}{|c|c|c|c|c|c|c|}
\hline & \multicolumn{2}{|c|}{$\begin{array}{l}\text { Intensive care unit } \\
\text { admission }\end{array}$} & \multicolumn{2}{|c|}{ Mechanical ventilation } & \multicolumn{2}{|r|}{ Death } \\
\hline & $\%$ & $\begin{array}{l}\text { Adjusted OR } \\
(95 \% \mathrm{Cl})\end{array}$ & $\%$ & $\begin{array}{c}\text { Adjusted OR } \\
(95 \% \mathrm{Cl})\end{array}$ & $\%$ & $\begin{array}{l}\text { Adjusted OR } \\
(95 \% \mathrm{Cl})\end{array}$ \\
\hline \multicolumn{7}{|c|}{ Hospitalization outcomes by hyperinflammation on $\mathrm{cHIS}^{1}$} \\
\hline cHIS <2 (n=112) & $21 \%$ & 1.0 (Ref) & $1 \%$ & 1.0 (Ref) & $3 \%$ & 1.0 (Ref) \\
\hline $\mathrm{cHIS} \geq 2(\mathrm{n}=177)$ & $48 \%$ & $3.5(2.0-6.0)$ & $37 \%$ & $66.2(9.0-487.8)$ & $27 \%$ & $16.4(4.8-56.4)$ \\
\hline \multicolumn{7}{|c|}{ Hospitalization outcomes by rheumatic disease status } \\
\hline $\begin{array}{l}\text { Comparators } \\
\qquad(n=232)\end{array}$ & $30 \%$ & 1.0 (Ref) & $19 \%$ & 1.0 (Ref) & $16 \%$ & 1.0 (Ref) \\
\hline $\begin{array}{l}\text { Rheumatic cases } \\
\quad(n=57)\end{array}$ & $51 \%$ & $1.87(1.03-3.40)$ & $39 \%$ & $2.46(1.30-4.67)$ & $21 \%$ & $1.32(0.61-2.88)$ \\
\hline
\end{tabular}

Matching factors: age, sex, and date of $+\mathrm{PCR} .{ }^{1}$ Adjusted for age, sex, and case status. ${ }^{2}$ Adjusted for race, smoking, comorbidities, and body mass index.cHIS, COVID19-associated hyperinflammation score; $\mathrm{Cl}$, confidence interval; OR, odds ratio; PCR polymerase chain reaction; SARS-CoV-2, severe acute respiratory syndrome coronavirus 2 .

Conclusion: Patients with systemic rheumatic disease hospitalized for COVID19 had higher risk for hyperinflammation, kidney injury, and mechanical ventilation than non-rheumatic comparators. We validated the cHIS in our cohort, which was strongly associated with poor COVID-19 outcomes. These findings highlight that hospitalized patients with rheumatic diseases may be vulnerable to poor COVID-19 outcomes.

REFERENCES:

[1] Webb BJ et al. Clinical criteria for COVID-19-associated hyperinflammatory syndrome. Lancet Rheumatol. 2020 Dec;2(12):e754-e763.
Figure:

Median peak laboratory values and composite COVID-19-associated hyperinflammation scores (cHIS) for systemic rheumatic disease cases $(n=57)$ and matched comparators $(n=232)$ during hospitalization for COVID-19. Error bars indicate interquartile range. cHIS was derived from 39,900 laboratory results, and the components are listed.
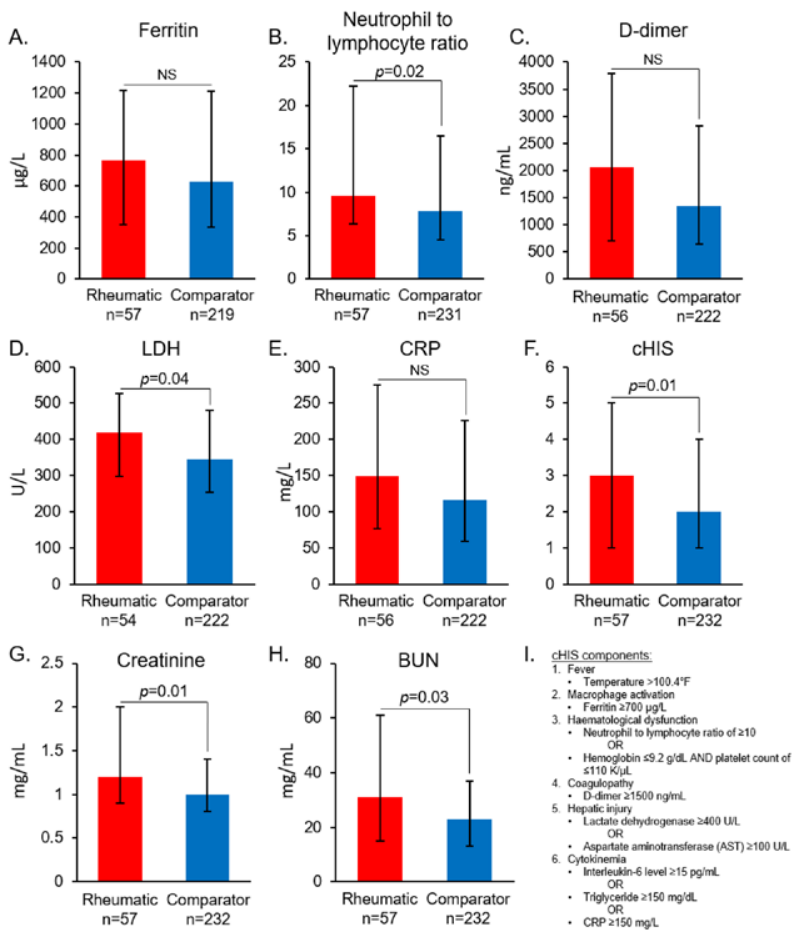

Disclosure of Interests: Tiffany Hsu: None declared, Kristin D'Silva: None declared, Naomi Serling-Boyd: None declared, Jiaqi Wang: None declared, Alisa Mueller: None declared, Xiaoqing Fu: None declared, Lauren Prisco: None declared, Lily Martin: None declared, Kathleen Vanni: None declared, Alessandra Zaccardelli: None declared, Claire Cook: None declared, Hyon Choi Consultant of: Dr. Choi reports consultancy fees from Takeda, Selecta, GlaxoSmithKline, and Horizon, Grant/research support from: Dr. Choi reports research support from AstraZeneca., Yuqing Zhang: None declared, Ellen Gravallese: None declared, Zachary Wallace Consultant of: Dr. Wallace reports consulting fees from Viela Bio and MedPace., Grant/research support from: Dr. Wallace reports research support from Bristol-Myers Squibb and Principia., Jeffrey Sparks Consultant of: Dr. Sparks reports consultancy fees from Bristol-Myers Squibb, Gilead, Inova, Janssen, Optum, and Pfizer., Grant/ research support from: Dr. Sparks reports research support from Amgen and Bristol-Myers Squibb.

DOI: 10.1136/annrheumdis-2021-eular.936

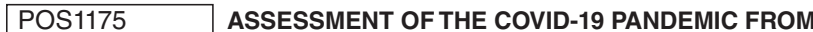 THE PERSPECTIVE OF PEOPLE WITH RHEUMATIC MUSCULOSKELETAL DISEASES IN EUROPE. RESULTS FROM THE REUMAVID STUDY (PHASE 1)}

M. Garrido-Cumbrera ${ }^{1}$, H. Marzo-Ortega ${ }^{2}$, J. Correa-Fernández ${ }^{1}$, S. SanzGómez ${ }^{1}$, L. Christen ${ }^{3}$, V. Navarro-Compán ${ }^{4}$ on behalf of REUMAVID working group. ${ }^{1}$ Universidad de Sevilla, Health \& Territory Research (HTR), Sevilla, Spain; ${ }^{2}$ University of Leeds, Leeds Institute for Rheumatic and Musculoskeletal Medicine, Leeds, United Kingdom; ${ }^{3}$ Novartis Pharma AG, Patient Engagement, Basel, Switzerland; ${ }^{4}$ Hospital Universitario La Paz, IdiPaz, Madrid, Spain

Background: The COVID-19 pandemic is an unprecedented public health crisis affecting people worldwide, including those with rheumatic and musculoskeletal diseases (RMDs). 